\title{
MODULARITY RELATIONS IN LATTICES
}

\section{R. J. MIHALEK}

1. Introduction. Linear independence has been formulated latticetheoretically by G. Birkhoff [1], J. von Neumann [4] and, in particular, L. R. Wilcox [5], who studied it in connection with ordinary modularity considered as a binary relation. In this work, the concept of a modularity relation is defined abstractly from which the theory of independence is developed. These results generalize those of S. Maeda [2] whose abstraction of independence characterizes ordinary independence. Also quasi-modularity relations are considered abstractly, which relations arise in the theory of quasi-dual-ideals [7]. Relations studied earlier by the author [3] are shown to be instances of the abstract relations considered here.

Throughout this paper $L$ is to be a lattice with order $\leqq$, join + and meet. For $b, c \in L,(b, c) M($ read $(b, c)$ modular) means $(a+b) c$ $=a+b c$ for every $a \leqq c$ ( $M$ will be referred to as ordinary modularity).

The notations $C,+, \cdot, \Theta, \times$ are respectively set-theoretic inclusion, sum, product, the empty set and cartesian product, and the set of all elements $x$ with the property $E(x)$ is denoted by $[x ; E(x)]$.

2. Modularity relations and independence. First, the notion of a modularity relation is defined abstractly, which is then used in the definition of the independence relation and the development of the independence theory.

(2.1) Definition. Let $R \subset T \subset L \times L$. The relation $R$ is a modularity relation under $T$ means

(a) $(b, c) R, b^{\prime} \leqq b, c^{\prime} \leqq c, b^{\prime} c^{\prime}=b c,\left(b^{\prime}, c^{\prime}\right) T$ implies $\left(b^{\prime}, c^{\prime}\right) R$;

(b) $(c, d) R,(b, c+d) R, b(c+d)=c d$ implies $(b+c, d) R,(b+c) d=c d$.

Part (a) of the definition would be too broad for the purposes considered here if the condition $\left(b^{\prime}, c^{\prime}\right) T$ were omitted from the hypotheses. The set $T$ is introduced merely to provide a control on the pairs that are eligible to be in $R$ and its role will become evident in the examples considered in the subsequent sections.

(2.2) Definition. For $R$ a modularity relation under $T, R$ is said

(a) to satisfy the intersection property if $(c, d) R,(b, c+d) R$, $b(c+d)=c d$ implies $(b+d)(c+d)=d$;

(b) to be symmetric at $a$, for $a \in L$, if $(b, c) R, b c=a$ implies $(c, b) R$.

Examples exist showing that a modularity relation does not necessarily satisfy these properties.

Presented to the Society, January 20, 1959; received by the editors April 14, 1959. 
(2.3) Definition. Let $R$ be a modularity relation under $T$. For $n \geqq 2, a, a_{1}, \cdots, a_{n} \in L,\left(a_{1}, \cdots, a_{n}\right) R_{a}\left(\operatorname{read}\left(a_{1}, \cdots, a_{n}\right) R\right.$-independent over $a)$ means $\left(\sum_{U} a_{i}, \sum_{V} a_{i}\right) R,\left(\sum_{U} a_{i}\right)\left(\sum_{V} a_{i}\right)=a$ for every nonempty $U, V \subset[1, \cdots, n]$ such that $j<k$ for $j \in U, k \in V$.

Throughout this section it is assumed that $R$ is a modularity relation under $T, n \geqq 2$ and $a, a_{1}, \cdots, a_{n} \in L$.

(2.4) Corollary. Let $\left(a_{1}, \cdots, a_{n}\right) R_{a}$.

(a) If $a_{i} \neq a$ for $1 \leqq i \leqq n$, then $a_{i} \neq a_{j}$ for $i \neq j$.

(b) If $1 \leqq k_{1}<\cdots<k_{m} \leqq n, m \geqq 2$, then $\left(a_{k_{1}}, \cdots, a_{k_{m}}\right) R_{a}$.

(c) If $a \leqq a_{i}^{\prime} \leqq a_{i}$ for $1 \leqq i \leqq n$, then $\left(a_{1}^{\prime}, \cdots, a_{n}^{\prime}\right) R_{a}$ provided $\left(\sum_{U} a_{i}^{\prime}, \sum_{V} a_{i}^{\prime}\right) T$ for every nonempty $U, V \subset[1, \cdots, n]$ such that $j<k$ for $j \in U, k \in V$.

(2.5) Theorem. If $\left(a_{1}, \cdots, a_{n}\right) R_{a}$, then $\left(a_{i}, a_{i+1}+\cdots+a_{n}\right) R_{a}$ for every $i=1, \cdots, n-1$, and conversely, provided $\left(a_{i}, \sum_{V} a_{j}\right) T$ for every nonempty $V \subset[i+1, \cdots, n]$.

PROOF. The forward implication is immediate. The reverse is obvious for $n=2$. Suppose it holds for $q \leqq n-1$ where $n \geqq 3$. Let $\left(a_{i}, a_{i+1}+\cdots+a_{n}\right) R_{a}$ for $i=1, \cdots, n-1$ and let $U, V \subset[1, \cdots, n]$ such that $U, V$ are nonempty and $j<k$ for $j \in U, k \in V$. Denote $U$ by $\left[j_{1}, \cdots, j_{u}\right]$ and $V$ by $\left[k_{1}, \cdots, k_{v}\right]$, where, without loss of generality, $j_{1}<\cdots<j_{u}<k_{1}<\cdots<k_{v}$. Then by (2.4.c),

$$
\left(a_{j_{i}}, a_{j_{i+1}}+\cdots+a_{k_{v}}\right) R_{a} \quad \text { for } i=1, \cdots, u
$$

and

$$
\left(a_{k_{i}}, a_{k_{i+1}}+\cdots+a_{k_{v}}\right) R_{a} \quad \text { for } i=1, \cdots, v-1 .
$$

In case $U+V \neq[1, \cdots, n]$, it follows from the induction hypothesis that $\left(a_{j_{1}}, \cdots, a_{j_{u}}, a_{k_{1}}, \cdots, a_{k_{v}}\right) R_{a}$, whence $\left(\sum_{U} a_{i}, \sum_{V} a_{i}\right) R_{a}$. Let $U+V=[1, \cdots, n]$. From the above argument,

$$
\left(a_{j_{2}}+\cdots+a_{j_{u}}, \sum_{V} a_{i}\right) R_{a}
$$

and by hypotheses, $\left(a_{j_{1}}, a_{j_{2}}+\cdots+a_{j_{u}}+\sum_{V} a_{i}\right) R_{a}$. Thus (2.1.b) yields $\left(\sum_{U} a_{i}, \sum_{V} a_{i}\right) R_{a}$. Hence the reverse implication holds for $q=n$ and the result follows by induction.

(2.6) THEOREM. Let $R$ satisfy the intersection property. If $\left(a_{1}, \cdots, a_{n}\right) R_{a}$, then $\left(\sum_{U} a_{i}\right)\left(\sum_{V} a_{i}\right)=\sum_{U V} a_{i}$ for every $U, V$ $\subset[1, \cdots, n]$ such that $U V \neq \Theta$ and $j<k<m$ for $j \in U-U V$, $k \in V-U V, m \in U V$.

Proof. Let $W=U-U V, X=V-U V$. Then by the hypotheses, 
$\left(\sum_{X} a_{i}, \quad \sum_{U V} a_{i}\right) R_{a}, \quad\left(\sum_{W} a_{i}, \quad \sum_{X} a_{i}+\sum_{U V} a_{i}\right) R_{a}, \quad$ whence $\left(\sum_{U} a_{i}\right)\left(\sum_{V} a_{i}\right)=\left(\sum_{W} a_{i}+\sum_{U V} a_{i}\right)\left(\sum_{X} a_{i}+\sum_{U V} a_{i}\right)=\sum_{U V} a_{i}$ by virtue of the intersection property.

(2.7) Lемма. Let $R$ satisfy the intersection property. If $\left(a_{1}, \cdots, a_{n}\right) R_{a}, U+V=[1, \cdots, n], U V=\Theta$, then $\left(\sum_{U} a_{i}\right)\left(\sum_{V} a_{i}\right)$ $=\left(a_{n}\right)\left(\sum_{U} a_{i}\right)\left(\sum_{V} a_{i}\right)$.

Proof. Let $U, V \neq \Theta$ and let $1 \in U$. Partition the set $[1, \cdots, n]$ with sets $W_{i}$ defined so that $W_{2 i-1} \subset U, W_{2 i} \subset V$, and $j^{\prime}<k^{\prime}$ for $j^{\prime} \in W_{j}, k^{\prime} \in W_{k}, j<k$. (The existence of such a partition is readily proved inductively.) Then $1 \in W_{1}$ and for some $m, n \in W_{m}$. The result is immediate for $m=2$; let $m \geqq 3$. Define $b_{j}=\sum_{w_{j}} a_{i}$ for $1 \leqq j \leqq m$. Then $\left(\sum_{V} a_{i}\right)\left(\sum_{V} a_{i}\right)=\left(\sum_{V} a_{i}\right)\left(b_{1}+\sum_{3}^{m} b_{i}\right)\left(b_{2}+\sum_{3}^{m} b_{i}\right)\left(\sum_{V} a_{i}\right)$ $=\left(\sum_{U} a_{i}\right)\left(\sum_{3}^{m} b_{i}\right)\left(\sum_{V} a_{i}\right)$, the last equality holding by virtue of the intersection property. For $m \geqq 4$, let $3 \leqq q<m$. Then

$$
\left(\sum_{1}^{q-1} b_{i}+\sum_{a+1}^{m} b_{i}\right) \geqq \sum_{U} a_{i}
$$

or $\sum_{V} a_{i}$ according as $q$ is even or odd. Thus

$$
\begin{aligned}
\left(\sum_{U} a_{i}\right)\left(\sum_{q}^{m} b_{i}\right) & \left(\sum_{V} a_{i}\right) \\
& =\left(\sum_{U} a_{i}\right)\left(\sum_{1}^{q-1} b_{i}+\sum_{q+1}^{m} b_{i}\right)\left(b_{q}+\sum_{q+1}^{m} b_{i}\right)\left(\sum_{V} a_{i}\right) \\
& =\left(\sum_{U} a_{i}\right)\left(\sum_{q+1}^{m} b_{i}\right)\left(\sum_{V} a_{i}\right) .
\end{aligned}
$$

Therefore

$$
\left(\sum_{U} a_{i}\right)\left(\sum_{V} a_{i}\right)=\left(\sum_{U} a_{i}\right)\left(b_{m}\right)\left(\sum_{V} a_{i}\right) .
$$

Let $X=W_{m}-[n]$ with $X \neq \Theta$; otherwise, the proof is complete. Then $\left(\sum_{1}^{m-1} b_{i}+a_{n}\right) \geqq \sum_{U} a_{i}$ or $\sum_{V} a_{i}$ according as $m$ is even or odd, whence

$$
\begin{aligned}
\left(\sum_{U} a_{i}\right)\left(b_{m}\right)( & \left.\sum_{V} a_{i}\right) \\
& =\left(\sum_{U} a_{i}\right)\left(\sum_{1}^{m-1} b_{i}+a_{n}\right)\left(\sum_{X} a_{i}+a_{n}\right)\left(\sum_{V} a_{i}\right) \\
& =\left(\sum_{U} a_{i}\right)\left(a_{n}\right)\left(\sum_{V} a_{i}\right) .
\end{aligned}
$$


Hence

$$
\left(\sum_{U} a_{i}\right)\left(\sum_{V} a_{i}\right)=\left(a_{n}\right)\left(\sum_{U} a_{i}\right)\left(\sum_{V} a_{i}\right) .
$$

(2.8) THEOREM. Let $R$ satisfy the intersection property. If $\left(a_{1}, \cdots, a_{n}\right) R_{a}$, then for nonempty disjoint $U, V \subset[1, \cdots, n]$, $\left(\sum_{U} a_{i}\right)\left(\sum_{v} a_{i}\right)=a$.

Proof. The result is immediate for $n=2$. Suppose it holds for $q \leqq n-1$ where $n \geqq 3$. Then it holds for $U+V \neq[1, \cdots, n]$, with an application of (2.4.b). Let $U+V=[1, \cdots, n]$. From the lemma, $\left(\sum_{U} a_{i}\right)\left(\sum_{V} a_{i}\right)=\left(a_{n}\right)\left(\sum_{U} a_{i}\right)\left(\sum_{V} a_{i}\right)$. Let $n \in V$. Then for $V=[n]$, $\left(\sum_{U} a_{i}\right)\left(\sum_{V} a_{i}\right)=a$ by definition, and for $V \neq[n],\left(\sum_{U} a_{i}\right)\left(\sum_{V} a_{i}\right)$ $=\left(a_{n}\right)\left(\sum_{U} a_{i}\right)\left(\sum_{V} a_{i}\right)=a \sum_{V} a_{i}=a$ by the induction hypothesis. Similarly, for $n \in U,\left(\sum_{U} a_{i}\right)\left(\sum_{V} a_{i}\right)=a$. Hence the result holds for $n=q$ and the proof is complete.

(2.9) Definition. Define $\left(a_{1}, \cdots, a_{n}\right) \bar{R}_{a}\left(\operatorname{read}\left(a_{1}, \cdots, a_{n}\right)\right.$ symmetrically $R$-independent over $a)$ to mean $\left(a_{i_{1}}, \cdots, a_{i_{n}}\right) R_{a}$ for every permutation $\left(i_{1}, \cdots, i_{n}\right)$ of the integers $[1, \cdots, n]$.

(2.10) CoRollary. (a) The relation $\bar{R}_{a}$ is symmetric. (b) If $\left(a_{1}, \cdots, a_{n}\right) \bar{R}_{a}$, then $\left(a_{1}, \cdots, a_{n}\right) R_{a}$.

(2.11) THEOREM. If $\left(a_{1}, \cdots, a_{n}\right) \bar{R}_{a}$, then $\left(a_{j}, \sum_{i \neq j} a_{i}\right) \bar{R}_{a}$ for $1 \leqq j \leqq n$, and conversely, provided $\left(a_{j}, \sum_{V} a_{i}\right) T$ for every nonempty $V \subset[1, \cdots, n]$ such that $j \notin V$.

Proof. This follows from (2.5) in a manner similar to the corresponding result in [5].

(2.12) THEOREM. Let $R$ satisfy the intersection property. If $\left(a_{1}, \cdots, a_{n}\right) \bar{R}_{a}$, then $\left(\sum_{U} a_{i}\right)\left(\sum_{V} a_{i}\right)=\sum_{U V} a_{i}$ for every $U, V$ $\subset[1, \cdots, n]$ such that $U V \neq \Theta$.

Proof. Let $U \nsubseteq V$ and $V \nsubseteq U$. Then let $U-U V=\left[i_{1}, \cdots, i_{u}\right]$, $V-U V=\left[j_{1}, \cdots, j_{v}\right], U V=\left[k_{1}, \cdots, k_{w}\right]$, where the $i_{m}, j_{m}$ and $k_{m}$ are distinct. Define

$$
b_{m}= \begin{cases}a_{i_{m}} & \text { for } 1 \leqq m \leqq u, \\ a_{j_{m-u}} & \text { for } u+1 \leqq m \leqq u+v, \\ a_{k_{m-u-v}} & \text { for } u+v+1 \leqq m \leqq u+v+w .\end{cases}
$$

Then $\left(b_{1}, \cdots, b_{u+v+w}\right) R_{a}$ by (2.9) and (2.4.b). Also

$$
U^{\prime}=[1, \cdots, u, u+v+1, \cdots, u+v+w]
$$


and

$$
V^{\prime}=[u+1, \cdots, u+v+w]
$$

satisfy the hypotheses of (2.6), whence

$$
\left(\sum_{U} a_{i}\right)\left(\sum_{V} a_{i}\right)=\left(\sum_{U^{\prime}} b_{i}\right)\left(\sum_{V^{\prime}} b_{i}\right)=\sum_{U^{\prime} V^{\prime}} b_{i}=\sum_{U V} a_{i} .
$$

In the remainder of this section, some results are stated for $R$ symmetric at $a$. The proofs of these results are similar to those of the corresponding results in [5] and will be omitted. In case $R$ were a symmetric relation, it is evident that $R$ would be symmetric at $a$ for every $a \in L$. If $R$ is symmetric at $a$, then the relation $R_{a}$ is symmetric, or equivalently, $(b, c) R_{a}$ if and only if $(b, c) \bar{R}_{a}$.

(2.13) Lemma. Let $R$ be symmetric at $a$. If $(c, b, d) R_{a}$, then $(b, c, d) R_{a}$.

(2.14) Theorem. If $R$ is symmetric at $a$, then $\left(a_{1}, \cdots, a_{n}\right) \bar{R}_{a}$ if and only if $\left(a_{1}, \cdots, a_{n}\right) R_{a}$.

(2.15) CoRollary. If $R$ is symmetric at $a$, then $\left(a_{1}, \cdots, a_{n}\right) R_{a}$ if and only if $\left(\sum_{U} a_{i}, \sum_{V} a_{i}\right) R_{a}$ for every nonempty disjoint $U, V$ $\subset[1, \cdots, n]$.

(2.16) Theorem. Let $R$ be symmetric at $a$ and let $b_{1}, \cdots, b_{m} \in L$ where $m \geqq 2$. If $\left(a_{1}, \cdots, a_{n}\right) R_{a},\left(b_{1}, \cdots, b_{m}\right) R_{a}$ and $\left(\sum_{1}^{n} a_{i}, \sum_{1}^{m} b_{i}\right) R_{a}$, then $\left(a_{1}, \cdots, a_{n}, b_{1}, \cdots, b_{m}\right) R_{a}$.

(2.17) Corollary. Let $R$ be symmetric at $a$ and for $j=1, \cdots, n$, let $m_{j} \geqq 2$ and $a_{i j} \in L$ for $i=1, \cdots, m_{j}$. If $\left(a_{1 j}, \cdots, a_{m_{j}}\right) R_{a}$ for $j=1, \cdots, n$ and if $\left(\sum_{1}^{m_{1}} a_{i 1}, \cdots, \sum_{1}^{m_{n}} a_{i n}\right) R_{a}$, then

$$
\left(a_{11}, \cdots, a_{m_{1} 1}, \cdots, a_{1 n}, \cdots, a_{m_{n} n}\right) R_{a} .
$$

3. Quasi-modularity relations. In the study of quasi-dual-ideals, the relations of weak modularity, as denoted by Wilcox [7], and quasi-modularity, as denoted by the author [3], arise with properties similar to those of ordinary modularity. In this section the material of $\$ 2$ is applied in an abstraction of these relations.

(3.1) Definition. A nonempty subset $S$ of $L$ is a quasi-dual-ideal (q.d.i.) if

(a) $x \in S, y \geqq x$ implies $y \in S$;

(b) $x, y \in S,(x, y) M$ implies $x y \in S$.

The smallest q.d.i. containing a set $T$ (or elements $a, b, c, \cdots$ ) is denoted by $\{T\}$ (or $\{a, b, c, \cdots\}$ ). The set of all q.d.i. is $\mathscr{L}$ and the set of all principal q.d.i. (of the form $\{a\}$ ) is $\delta$. For $\alpha, \beta \in \mathcal{L}$, 
$\alpha \leqq \beta$ means $\alpha \supset \beta, \alpha \cup \beta=\alpha \cdot \beta$ and $\alpha \cap \beta=\{\alpha+\beta\}$.

It is useful to note that the principal q.d.i. of $L$ coincide with the principal dual ideals of $L$. For the next corollary and for all statements with reference to $\mathscr{L}$ in the remainder of the paper, it is assumed that l.u.b. $L=1$ exists.

(3.2) CoROllaRy. The set $\&$ is a complete lattice with respect to $\leqq$; the lattice operations are $\cup, \cap$, and $L$ and $\{1\}$ are the zero and unit respectively. If $(b, c) M,\{b, c\}=\{b c\}$. The lattice $L$ is isomorphic to the set $\mathcal{S}$, a lattice subset (not necessarily a sublattice) of $\mathcal{L}$, under $a \rightarrow\{a\}$.

Proof. In s, l.u.b. $[\{a\},\{b\}]=\{a+b\}$ and g.l.b. $[\{a\},\{b\}]$ $=\{a b\}$. The isomorphism now follows and the remainder is immediate.

(3.3) Definition. Let $Q \subset L \times L$. Then $Q$ is a quasi-modularity relation means that $Q=[(\{b\},\{c\}) ;(b, c) Q]$ is a modularity relation under $\mathcal{S} \times \mathcal{S}$ in $\mathcal{L}$. For $Q$ a quasi-modularity relation, $Q$ is said to satisfy the intersection property (to be symmetric at $\alpha$, for $\alpha \in \mathfrak{L}$ ) if $Q$ satisfies the intersection property (if $Q$ is symmetric at $\alpha$ ) in $\&$.

(3.4) Definition. Let $Q$ be a quasi-modularity relation. For $n \geqq 2, a_{1}, \cdots, a_{n} \in L$ and $\alpha \in \mathcal{L},\left(a_{1}, \cdots, a_{n}\right) Q_{\alpha}\left(\operatorname{read}\left(a_{1}, \cdots, a_{n}\right)\right.$ $Q$-quasi-independent over $\alpha)$ means $\left(\left\{a_{1}\right\}, \cdots,\left\{a_{n}\right\}\right) Q_{\alpha}$ where $\mathcal{Q}$ is defined as in (3.3).

(3.5) Corollary. If $\left(a_{1}, \cdots, a_{n}\right) Q_{\alpha}$, then $\left(\sum_{U} a_{i}, \sum_{V} a_{i}\right) Q$, $\left\{\sum_{U} a_{i}, \sum_{V} a_{i}\right\}=\alpha$ for every nonempty $U, V \subset[1, \cdots, n]$ such that $j<k$ for $j \in U, k \in V$, and conversely.

The corollary shows the analogy between $Q$-quasi-independence over a q.d.i. of $L$ and $R$-independence over an element of $L$ as defined in (2.3). The results of the independence theory of the previous section may be applied to $Q$, yielding a corresponding theory for $Q$. If one keeps in mind the equalities $\{b\} \cup\{c\}=\{b+c\},\{b\} \cap\{c\}$ $=\{b, c\}$ and that $\alpha \leqq\{a\}$ means $a \in \alpha$, the independence theory for $Q$ may be stated free of the notation of the lattice $\mathcal{L}$.

4. Examples. An example of a modularity relation is obtained from a special case of relative modularity, the latter being a relativization of ordinary modularity.

(4.1) Definition. For $S \subset L, b, c \in L,(b, c) M_{S}(\operatorname{read}(b, c)$ modular relative to $S)$ means $(a+b) c=a+b c$ for every $a \in S$ such that $a \leqq c$.

Evidently, $M=M_{L}$. In addition, $M_{S}$ satisfies many of the properties of $M$, some in a modified form. In particular, the next lemma is of interest. 
(4.2) Lemma. If $(b, c) M_{S}, b^{\prime} \leqq b, c^{\prime} \leqq c, b^{\prime} c^{\prime}=b c$, then $\left(b^{\prime}, c^{\prime}\right) M_{S}$.

Proof. Let $a \leqq c^{\prime}, a \in S$. Then $\left(a+b^{\prime}\right) c^{\prime} \leqq(a+b) c=a+b c=a+b^{\prime} c^{\prime}$, whence $\left(b^{\prime}, c^{\prime}\right) M_{S}$ since the reverse inequality $\left(a+b^{\prime}\right) c^{\prime} \geqq a+b^{\prime} c^{\prime}$ holds universally for $a \leqq c^{\prime}$.

(4.3) THEOREM. If $S$ is join-closed, then $R=(S \times L) \cdot M_{S}$ is a modularity relation under $S \times L$.

Proof. Part (a) of (2.1) readily follows with an application of (4.2). For Part (b), let $(c, d) R,(b, c+d) R, b(c+d)=c d$. Then $b, c \in S$, $(b+c, d) \in S \times L$ and $b(c+d) \leqq c$. Now let $a \leqq d, a \in S$. Then $a+c \in S$, $a+c \leqq c+d$ and

$$
\begin{aligned}
(a+(b+c)) d & =((a+c)+b)(c+d) d=((a+c)+b(c+d)) d \\
& =(a+(c+b(c+d))) d=(a+c) d \\
& =a+c d \leqq a+(b+c) d .
\end{aligned}
$$

Thus $(b+c, d) M_{S}$, whence $(b+c, d) R$. Also

$$
(b+c) d=(c+b)(c+d) d=(c+b(c+d)) d=c d .
$$

(4.4) Theorem. If $S$ is join-closed, then $R=(S \times S) \cdot M_{S}$ is a modularity relation under $S \times S$ satisfying the intersection property.

Proof. The proof that $R$ is a modularity relation under $S \times S$ is essentially the proof of (4.3). For the remainder, let $(c, d) R,(b, c+d) R$, $b(c+d)=c d$. Then $d \in S, b(c+d) \leqq d$ and since $(b, c+d) M_{S},(b+d)$ $\cdot(c+d)=d+b(c+d)=d$.

Two examples of quasi-modularity relations are now considered.

(4.5) Definition. For $b, c \in L$,

(a) $(b, c) M_{0}($ read $(b, c)$ weakly modular) means $\{a+b, c\}=\{a\}$ $\cup\{b, c\}$ for every $a \leqq c$;

(b) $(b, c) M_{q}$ (read $(b, c)$ quasi-modular) means $(b, c) M_{S}$ where $S=\{b, c\}$.

(4.6) Theorem. The relations $M_{0}$ and $M_{q}$ are quasi-modularity relations satisfying the intersection property.

The proof of this theorem is omitted. It is of interest to note that always $M_{0} \subset M_{q}$ and that examples of left-complemented [6] lattices exist for which the inclusion is proper.

To show that the notion of a modularity relation is more general than ordinary modularity, one may consider the relation $Q$ in $\mathscr{L}$ corresponding to $M_{0}$, which is incidentally $(\delta \times S) \cdot M_{\mathcal{S}}$. In case $L$ is not a modular lattice, this $Q$, although a modularity relation, is not ordinary modularity for $\mathscr{L}$. 


\section{REFERENCES}

1. G. Birkhoff, Abstract linear independence and lattices, Amer. J. Math. vol. 57 (1935) pp. 800-804.

2. S. Maeda, Dimension functions on certain general lattices, J. Sci. Hiroshima Univ. Ser. A vol. 19 (1955) pp. 211-237.

3. R. J. Mihalek, Modularity relations in lattices. Preliminary report, Notices Amer. Math. Soc. vol. 5 (1958) p. 349.

4. J. von Neumann, Lectures on continuous geometries, vol. 1, Princeton, 1936.

5. L. R. Wilcox, Modularity in the theory of lattices, Ann. of Math. vol. 40 (1939) pp. $490-505$.

6. - A note on complementation in lattices, Bull. Amer. Math. Soc. vol. 48 (1942) pp. 453-458.

7. - Modular extensions of semi-modular lattices, Bull. Amer. Math. Soc. vol. 61 (1955) p. 524.

ILLINOIS INSTITUTE OF TECHNOLOGY 